\title{
Magnetohydrodynamics and Radiation Effects on Unsteady Convection Flow of Micropolar Fluid Past a Vertical Porous Plate with Variable Wall Heat Flux
}

\author{
M. Gnaneswara Reddy \\ Department of Mathematics, Acharya Nagarjuna University Ongole Campus, Ongole 523 001, AP, India \\ Correspondence should be addressed to M. Gnaneswara Reddy, mgrsvu@gmail.com
}

Received 28 March 2012; Accepted 19 April 2012

Academic Editors: J. K. Brennan, A. Ghoufi, and H. Hirao

Copyright (C) 2012 M. Gnaneswara Reddy. This is an open access article distributed under the Creative Commons Attribution License, which permits unrestricted use, distribution, and reproduction in any medium, provided the original work is properly cited.

An analysis is presented for the problem of the unsteady two-dimensional laminar flow of a viscous incompressible micropolar fluid past a vertical porous plate in the presence of a transverse magnetic field and thermal radiation with variable heat flux. The free stream velocity follows an exponentially increasing or decreasing small perturbation law. A uniform magnetic field acts perpendicularly to the porous surface in which it absorbs the micropolar fluid with a suction velocity varying with time. The Rosseland approximation is used to describe radiative heat transfer in the limit of optically thick fluids. The effects of flow parameters and thermophysical properties on the flow temperature fields across the boundary layer are investigated. The method of solution can be applied for small perturbation approximation. Numerical results of velocity profiles of micropolar fluids are compared with the corresponding flow problems for a Newtonian fluid. Also, the results of the skin-friction coefficient and the couple stress coefficient at the wall are prepared with various values of the fluid properties.

\section{Introduction}

The classical Navier-Stokes theory does not describe adequately the flow properties of polymeric fluids, colloidal suspensions, and fluids containing certain additives. Eringen [1] proposed the theory of micropolar fluids which shows microrotation effects as well as microinertia. The theory of thermomicropolar fluids was developed by Eringen [2] by extending his theory of micropolar fluid. Physically, they represent fluids consisting of randomly oriented particles suspended in a viscous medium. The flow characteristics of the boundary layer of micropolar fluid over a semi-infinite plate in different situations have been studied by many authors in (Ahmadi [3], Peddieson and McNitt [4], Takhar and Soundagekhar [5], and Gorla [6]). The study of the flow and heat transfer for a micropolar fluid past porous plate embedded in a porous medium has attracted the interest of many investigators in view of its applications in many engineering problems such as oil exploration, chemical catalytic reactors, thermal insulation, and geothermal energy extractions (Raptis [7]). Sharma and Gupta [8] considered thermal convection in micropolar fluids in porous medium. Kim [9] presented an analysis of an unsteady convection flow of a micropolar fluid past a vertical porous plate embedded in a porous medium.

The study of magnetohydrodynamics (MHD) plays an important role in agriculture, engineering, and petroleum industries. The problem of free convection under the influence of a magnetic field has attracted the interest of many researchers in view of its applications in geophysics and astrophysics. In addition, this type of flow has attracted the interest of many investigators in view of its applications in many engineering problems such as MHD generators, plasma studies, nuclear reactors, and geothermal energy extractions. Kim [10] studied unsteady MHD convection flow of polar fluids past a semi-infinite vertical-moving porous plate in a porous medium. Soundalgekar [11] obtained approximate solutions for the two-dimensional flow of an incompressible, viscous fluid past an infinite porous vertical plate with constant suction velocity normal to the plate, 
the difference between the temperature of the plate and the free stream is moderately large causing the free convection currents. Raptis [12] studied mathematically the case of unsteady two-dimensional natural convective heat transfer of an incompressible, electrically conducting viscous fluid via a highly porous medium bound by an infinite vertical porous plate. El-Hakiem et al. [13] studied the effect of viscous and Joule heating on MHD-free convection flow with variable plate temperature in a micropolar fluid in the presence of uniform transverse magnetic field using the Kellerbox implicit scheme. Rahman and Sattar [14] analyzed magnetohydrodynamic convective flow of a micropolar fluid past a continuously moving vertical porous plate in the presence of heat generation/absorption.

In the context of space technology and in the processes involving high temperatures, the effects of radiation are of vital importance. Recent developments in hypersonic flights, missile reentry, rocket combustion chambers, power plants for interplanetary flight, and gas-cooled nuclear reactors, have focused attention on thermal radiation as a mode of energy transfer and emphasized the need for improved understanding of radiative transfer in these processes. Raptis [15] studied the flow of a micropolar fluid past a continuous moving plate by the presence of radiation. Abo-Eldahab and Ghonaim [16] analyzed the radiation effects on heat transfer of a micropolar fluid through a porous medium. Kim and Fedorov [17] considered the case of mixed convection flow of a micropolar fluid past a semi-infinite moving vertical porous plate with varying suction velocity normal to the plate in the presence of radiation.

The purpose of this work is to study radiation effects on unsteady Magnetohydrodynamics (MHD) mixed convection flow of micropolar fluid with variable heat flux has received a little attention. We also consider the free stream to consist of a mean velocity over which is superimposed an exponentially varying with time. The effects of various governing parameters on the velocity, microrotation and temperature, skinfriction coefficient, and the wall couple stress coefficient are shown in figures and table and discussed in detail.

\section{Mathematical Analysis}

An unsteady two-dimensional mixed convection flow of a laminar incompressible micropolar fluid past a vertical porous plate in the presence of a transverse magnetic field and thermal radiation with variable heat flux is considered. The $x^{*}$-axis is taken along the vertical porous plate in an upward direction and $y^{*}$-axis is taken normal to the plate. The acceleration of gravity $g$ is in a direction opposite to $x^{*}$-coordinate. The transversely applied magnetic field and magnetic Reynolds number are very small and hence the induced magnetic field is negligible. Viscous and Darcy resistance terms are taken into account as the constant permeability porous medium. It is also assumed here that the size of holes in the porous plate is significantly larger than a characteristic microscopic length scale of the micropolar fluid to simplify formulation of the boundary conditions. Due to the assumption of infinite plate, the flow variables are functions of $y^{*}$ and the time $t^{*}$ only. It is also assumed that the free stream to consist of a mean velocity and temperature over which are superimposed an exponentially varying with time. Under the usual Boussenesq's approximation, the equation of continuity, linear momentum, microrotation, and energy can be written as

continuity equation:

$$
\frac{\partial v^{*}}{\partial y^{*}}=0
$$

linear momentum equation:

$$
\begin{aligned}
\frac{\partial u^{*}}{\partial t^{*}}+v^{*} \frac{\partial u^{*}}{\partial y^{*}}= & -\frac{1}{\rho} \frac{\partial p^{*}}{\partial x^{*}}+\left(\nu+v_{r}\right) \frac{\partial^{2} u^{*}}{\partial y^{* 2}}+g \beta_{f}\left(T-T_{\infty}\right) \\
& -\frac{v}{K^{*}} u^{*}-\frac{\sigma B_{0}^{2}}{\rho} u^{*}+2 v_{r} \frac{\partial \omega^{*}}{\partial y^{*}}
\end{aligned}
$$

angular momentum equation:

$$
\rho j^{*}\left(\frac{\partial \omega^{*}}{\partial t^{*}}+v^{*} \frac{\partial \omega^{*}}{\partial y^{*}}\right)=\gamma \frac{\partial^{2} \omega^{*}}{\partial y^{* 2}},
$$

energy equation:

$$
\frac{\partial T}{\partial t^{*}}+v^{*} \frac{\partial T}{\partial y^{*}}=\alpha\left(\frac{\partial^{2} T}{\partial y^{* 2}}-\frac{1}{k} \frac{\partial q_{r}}{\partial y^{*}}\right) .
$$

The third term on the right-hand side of the momentum equation (2) denotes thermal buoyancy effect.

By using the Rosseland approximation, Brewster [18], the radiative heat flux in the $y^{*}$ direction is given by

$$
q_{r}=-\frac{4 \sigma_{s} \partial T^{4}}{3 k_{e} \partial y^{*}}
$$

where $\sigma_{s}$ is the Stefan-Boltzmann constant and $k_{e}$ is the mean absorption coefficient, respectively.

It should be noted that by Rosseland approximation, we limit our analysis to optically thick fluids. If the temperature differences within the flow are sufficiently small, then (4) can be linearized by expanding $T^{4}$ into the Taylor series about $T_{\infty}$ and neglecting higher terms to take the form:

$$
T^{4} \cong 4 T_{\infty}^{3} T-3 T_{\infty}^{4} .
$$

It is assumed that the porous plate moves with constant velocity in the longitudinal direction, and the free steam velocity follows an exponentially increasing or decreasing small perturbation law. It is also assumed that the plate temperature and suction velocity vary exponentially with time.

Under these assumptions, the appropriate boundary conditions for the velocity, microrotation, temperature, and concentration fields are

$$
\begin{gathered}
u^{*}=0, \quad \omega^{*}=-\frac{1}{2} \frac{\partial u^{*}}{\partial y^{*}}, \\
\frac{\partial T}{\partial y^{*}}=-\frac{q_{w}}{k}\left(1+\varepsilon B e^{n^{*} t^{*}}\right) \quad \text { at } y^{*}=0, \\
u^{*} \longrightarrow U_{\infty}^{*}=U_{0}\left(1+\varepsilon e^{n^{*} t^{*}}\right), \quad \omega^{*} \longrightarrow 0, \\
T \longrightarrow T_{\infty} \quad \text { as } y^{*} \longrightarrow \infty,
\end{gathered}
$$


where $B$ is a real positive constant and $\varepsilon$ is a small parameter such that $\varepsilon B \leq 1, T_{\infty}$ is the free stream dimensional temperature, $n^{*}$ is constant, $\varepsilon$ is small less than unity and $U_{0}$ is a scale of free steam velocity. The boundary condition for microrotation variable $\omega^{*}$ describes its relationship with the surface stress.

It is clear from (1) that the suction velocity normal to the plate is either a constant or a function of time. Hence it is assumed in the form:

$$
v^{*}=-V_{0}\left(1+\varepsilon A e^{n^{*} t^{*}}\right),
$$

where $A$ is a real constant, $\varepsilon$ and $\varepsilon A$ are small less than unity, and $V_{0}$ is the scale of suction velocity which is a nonzero positive constant. The negative sign indicates that the suction is towards the plate. Outside the boundary layer, (2) gives

$$
-\frac{1}{\rho} \frac{d p^{*}}{d x^{*}}=\frac{d U_{\infty}^{*}}{d t^{*}}+\frac{\nu}{K^{*}} U_{\infty}^{*}+\frac{\sigma B_{0}^{2}}{\rho} U_{\infty}^{*}
$$

In order to write the governing equations and the boundary conditions dimensionless form, the following nondimensional quantities are introduced

$$
\begin{gathered}
u=\frac{u^{*}}{U_{0}}, \quad v=\frac{v^{*}}{V_{0}}, \quad y=\frac{V_{0} y^{*}}{v}, \\
U_{\infty}=\frac{U_{\infty}^{*}}{U_{0}}, \quad \omega=\frac{v}{U_{0} V_{0}} \omega^{*}, \quad t=\frac{t^{*} V_{0}^{2}}{v}, \\
\theta=\frac{\left(T-T_{\infty}\right) k V_{0}}{\left(T_{w}-T_{\infty}\right) q_{w}}, \quad j=\frac{V_{0}^{2}}{v^{2}} j^{*}, \\
n=\frac{n^{*} V_{0}^{2}}{\nu}, \quad \mathrm{Gr}=\frac{\nu \beta_{f} g\left(T_{w}-T_{\infty}\right)}{U_{0} V_{0}^{2}}, \\
\operatorname{Pr}=\frac{\nu \rho C_{p}}{k}=\frac{\nu}{\alpha}=\frac{\mu C_{p}}{k}, \quad R=\frac{k k_{e}}{4 \sigma_{s} T_{\infty}^{3}} .
\end{gathered}
$$

Furthermore, the spin-gradient viscosity $\gamma$, which gives some relationship between the coefficients of viscosity and microinertia, is defined as

$$
\gamma=\left(\mu+\frac{\Lambda}{2}\right) j^{*}=\mu j^{*}\left(1+\frac{1}{2} \beta\right), \quad \beta=\frac{\Lambda}{\mu} .
$$

In view of (5), (6) and (9)-(13), the governing equations (2)(4) reduce to the following nondimensional form:

$$
\begin{aligned}
\frac{\partial u}{\partial t}-\left(1+\varepsilon A e^{n t}\right) \frac{\partial u}{\partial y}= & \frac{d U_{\infty}}{d t}+(1+\beta) \frac{\partial^{2} u}{\partial y^{2}} \\
+\operatorname{Gr} \theta+N\left(U_{\infty}-u\right)+2 \beta \frac{\partial \omega}{\partial y} & \\
\frac{\partial \omega}{\partial t}-\left(1+\varepsilon A e^{n t}\right) \frac{\partial \omega}{\partial y}= & \frac{1}{\eta} \frac{\partial^{2} \omega}{\partial y^{2}} \\
\frac{\partial \theta}{\partial t}-\left(1+\varepsilon A e^{n t}\right) \frac{\partial \theta}{\partial y} & =\frac{1}{\Gamma} \frac{\partial^{2} \theta}{\partial y^{2}}
\end{aligned}
$$

where $\eta=\mu j^{*} / \gamma=2 /(2+\beta), N=(M+(1 / K))$, and $\Gamma=$ $(1-(4 /(3 R+4))) \operatorname{Pr}$.
The boundary conditions (7) are then given by the following dimensionless equations:

$$
\begin{gathered}
u=0, \quad \frac{\partial \theta}{\partial y}=-\left(1+\varepsilon B e^{n t}\right), \quad \omega=-\frac{1}{2} \frac{\partial u}{\partial y} \text { at } y=0, \\
u \longrightarrow U_{\infty}, \quad \theta \longrightarrow 0, \quad \omega \longrightarrow 0 \text { as } y \longrightarrow \infty .
\end{gathered}
$$

\section{Solution of the Problem}

In order to reduce the above system of partial differential equations to a system of ordinary differential equations in dimensionless form, we perform an asymptotic analysis by representing the linear velocity, microrotation, and temperature in the neighborhood of the porous plate as

$$
\begin{aligned}
& u=u_{0}(y)+\varepsilon e^{n t} u_{1}(y)+O\left(\varepsilon^{2}\right)+\cdots, \\
& \omega=\omega_{0}(y)+\varepsilon e^{n t} \omega_{1}(y)+O\left(\varepsilon^{2}\right)+\cdots, \\
& \theta=\theta_{0}(y)+\varepsilon e^{n t} \theta_{1}(y)+O\left(\varepsilon^{2}\right)+\cdots .
\end{aligned}
$$

Substituting (16) into (14), and equating the harmonic and nonharmonic terms, and neglecting the higher-order terms of $\mathrm{O}\left(\varepsilon^{2}\right)$, we obtain the following pairs of equations for $\left(u_{0}, \omega_{0}, \theta_{0}\right)$ and $\left(u_{1}, \omega_{1}, \theta_{1}\right)$ :

$$
\begin{gathered}
(1+\beta) u_{0}^{\prime \prime}+u_{0}^{\prime}-N u_{0}=-N-\mathrm{Gr} \theta_{0}-2 \beta \omega_{0}^{\prime}, \\
(1+\beta) u_{1}^{\prime \prime}+u_{1}^{\prime}-(N+n) u_{1}=-(N+n) \\
-A u_{0}^{\prime}-\mathrm{Gr} \theta_{1}-2 \beta \omega_{1}^{\prime}, \\
\omega_{0}^{\prime \prime}+\eta \omega_{0}^{\prime}=0, \\
\omega_{1}^{\prime \prime}+\eta \omega_{1}^{\prime}-n \eta \omega_{1}=-A \eta \omega_{0}^{\prime}, \quad \theta_{0}^{\prime \prime}+\Gamma \theta_{0}^{\prime}=0, \\
\theta_{1}^{\prime \prime}+\Gamma \theta_{1}^{\prime}-n \Gamma \theta_{1}=-A \Gamma \theta_{0}^{\prime} .
\end{gathered}
$$

In the above equations, prime indicates differentiation with respect to $y$ only.

The corresponding boundary conditions can be written as

$$
\begin{gathered}
u_{0}=0, \quad u_{1}=0, \quad \omega_{0}=-\frac{1}{2} u_{0}^{\prime}, \quad \omega_{1}=-\frac{1}{2} u_{1}^{\prime}, \\
\theta_{0}^{\prime}=-1, \quad \theta_{1}^{\prime}=-B \quad \text { at } y=0, \\
u_{0}=1, \quad u_{1}=1, \quad \omega_{0} \longrightarrow 0, \quad \omega_{1} \longrightarrow 0, \\
\theta_{0} \longrightarrow 0, \quad \theta_{1} \longrightarrow 0, \quad \text { as } y \longrightarrow \infty .
\end{gathered}
$$


Solving (17) under the boundary conditions (18), we get

$$
\begin{aligned}
& u_{0}(y)=1+a_{1} e^{-h_{2} y}+a_{2} e^{-\Gamma y}+a_{3} e^{-\eta y}, \\
& u_{1}(y)=1+b_{1} e^{-h_{1} y}+b_{2} e^{-h_{2} y}+b_{3} e^{-h_{3} y} \\
& +b_{4} e^{-h_{4} y}+b_{5} e^{-\Gamma y}+b_{6} e^{-\eta y}, \\
& \omega_{0}(y)=c_{1} e^{-\eta y}, \\
& \omega_{1}(y)=c_{2} e^{-h_{1} y}-\frac{A \eta}{n} c_{1} e^{-\eta y}, \quad \theta_{0}(y)=\frac{1}{\Gamma} e^{-\Gamma y} \\
& \theta_{1}(y)=\frac{1}{h_{4}}\left(B+\frac{A \Gamma}{n}\right) e^{-h_{4} y}-\frac{A}{n} e^{-\Gamma y},
\end{aligned}
$$

where the exponential indices and coefficients are given in the appendix.

By virtue of (16), we obtain the velocity, microrotation, and temperature profiles as follows:

$$
\begin{gathered}
u(y, t)=1+a_{1} e^{-h_{2} y}+a_{2} e^{-\Gamma y}+a_{3} e^{-\eta y} \\
+\varepsilon e^{\delta t}\left(1+b_{1} e^{-h_{1} y}+b_{2} e^{-h_{2} y}+b_{3} e^{-h_{3} y}\right. \\
\left.+b_{4} e^{-h_{4} y}+b_{5} e^{-\Gamma y}+b_{6} e^{-\eta y}\right), \\
\omega(y, t)=c_{1} e^{-\eta y}+\varepsilon e^{\delta t}\left(c_{2} e^{-h_{1} y}-\frac{A \eta}{n} c_{1} e^{-\eta y}\right), \\
\theta(y, t)=\frac{1}{\Gamma} e^{-\Gamma y}+\varepsilon e^{\delta t}\left(\frac{1}{h_{4}}\left(B+\frac{A \Gamma}{n}\right) e^{-h_{4} y}-\frac{A}{n} e^{-\Gamma y}\right) .
\end{gathered}
$$

Knowing the velocity field in the boundary layer, we can calculate the skin-friction coefficient $C_{f}$ at the porous plate, which in the nondimensional form is given by

$$
\begin{aligned}
\tau_{w}^{*}= & \left.\mu \frac{\partial u^{*}}{\partial y^{*}}\right|_{y^{*}=0}, \\
C_{f}= & \frac{\tau_{w}^{*}}{\rho U_{0} V_{0}}=\left.\frac{\partial u}{\partial y}\right|_{y=0}=-\left(a_{1} h_{1}+a_{2} \Gamma+a_{3} \eta\right) \\
& -\varepsilon e^{n t}\left(b_{1} h_{1}+b_{2} h_{2}+b_{3} h_{3}+b_{4} h_{4}+b_{5} \Gamma+b_{6} \eta\right) .
\end{aligned}
$$

Knowing the microrotation in the boundary layer, we can calculate the couple stress coefficient $C_{m}$ at the porous plate, which in the nondimensional form is given by

$$
\begin{gathered}
M_{w}=\left.\gamma \frac{\partial w^{*}}{\partial y^{*}}\right|_{y^{*}=0}, \\
C_{m}=\frac{M_{w}}{\mu j U_{0}}=\left.\frac{\partial w}{\partial y}\right|_{y=0}=1+\varepsilon e^{n t}\left(\left(B+\frac{A \Gamma}{n}\right)-\frac{A \Gamma}{n}\right) .
\end{gathered}
$$

\section{Results and Discussion}

The formulation of the problem that accounts for the effects of MHD and radiation on flow of an incompressible, micropolar fluid along a vertical porous plate has been carried out in the preceding sections. This enables us to carry out the numerical computations for the velocity, microrotation, and temperature for various values of the flow and material parameters. In the present study the following default parameter values are adopted for computations: $\beta=0.2$, $t=1.0, n=0.1, \varepsilon=0.001, A=0.5, \mathrm{Gr}=2.0, M=2.0, K=$ $1.0, \operatorname{Pr}=0.71, R=2.0$, and $B=0.1$. All graphs therefore correspond to these values unless specifically indicated on the appropriate graph. Also, the boundary condition for $y \rightarrow \infty$ is replaced by where $y_{\max }$ is a sufficiently large value of $y$ where the velocity profile $u$ approaches the relevant free stream velocity. We choose $y_{\max }=8$ and a step size $\Delta y=0.001$.

The effect of viscosity ratio $\beta$ on the translational velocity and microrotation profiles across the boundary layer are presented in Figure 1. It is noteworthy that the velocity distribution is greater for a Newtonian fluid $(\beta=0)$ with given parameters, as compared with micropolar fluids until its peak value reaches. The translational velocity shows a decelerating nature near the porous plate as $\beta$-parameter increases, and then decays to the relevant free stream velocity. In addition, the magnitude of microrotation at the wall is decreased as $\beta$-parameter increases. However, the distributions of microrotation across the boundary layer do not show consistent variations with increment of $\beta$ parameter.

The translational velocity and the microrotation profiles against spanwise coordinate $y$ for different values of Grashof number Gr are described in Figure 2. The thermal Grashof number signifies the relative effect of the thermal buoyancy force to the viscous hydrodynamic force. It is observed that an increase in Gr leads to a rise in the values of velocity, but decreases due to microrotation. Here the positive values of $\mathrm{Gr}$ corresponds to a cooling of the surface by natural convection. In addition, the curves show that the peak value of velocity increases rapidly near the wall of the porous plate as $\mathrm{Gr}$ increases, and then decays to the free stream velocity.

For different values of the magnetic field parameter $M$, the translational velocity and microrotation profiles are plotted in Figure 3. It is obvious that the effect of increasing values of $M$-parameter results in a decreasing velocity distribution across the boundary layer. The results also show that the magnitude of microrotation on the porous plate is decreased as $M$-parameter increases.

For various values of the permeability parameter $K$, the profiles of the translational velocity and the microrotation across the boundary layer are shown in Figure 4. Clearly as $K$ increases, although the corresponding profiles of the microrotation is difficult to show clearly due to very little variation, the translational velocity and the microrotation profiles tend to increase.

Figure 5 shows the translational velocity and the temperature profiles across the boundary layer for different values of Prandtl number Pr. The Prandtl number defines the ratio of momentum diffusivity to thermal diffusivity. The results show the effect of increasing values Pr on decreasing the translational velocity at the wall and then approach to the free stream boundary layer conditions. Typical variations of the temperature profiles along the spanwise coordinate $y$ are 


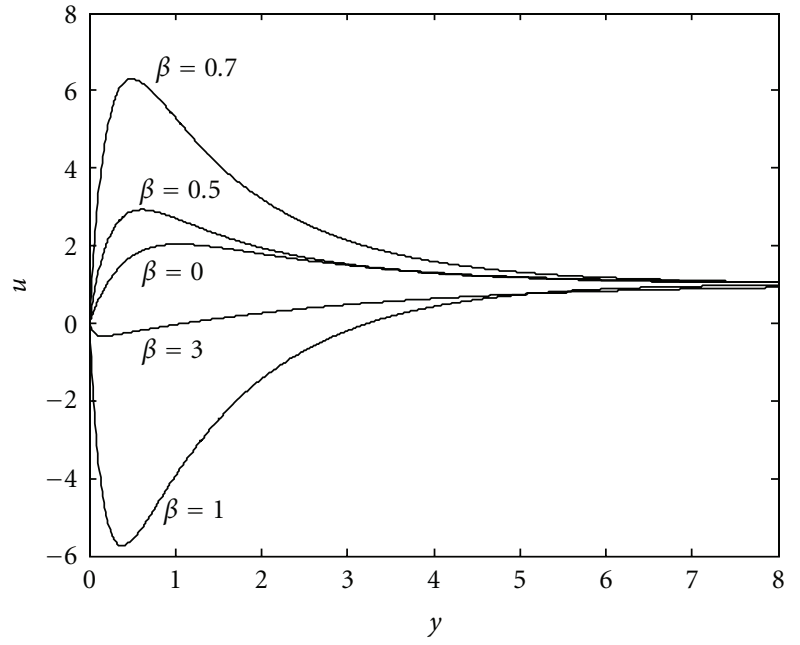

(a)

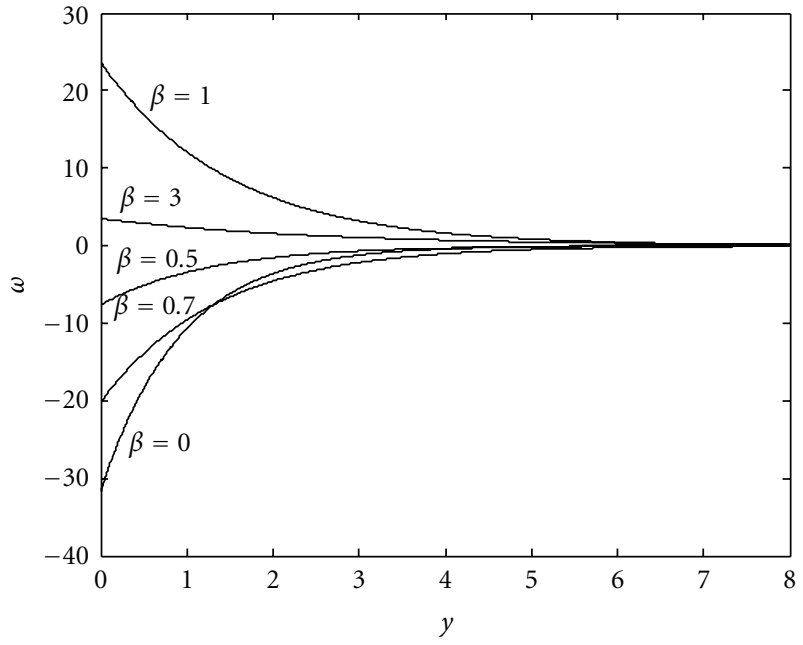

(b)

FigURE 1: Velocity and microrotation profiles for various values of $\beta$.

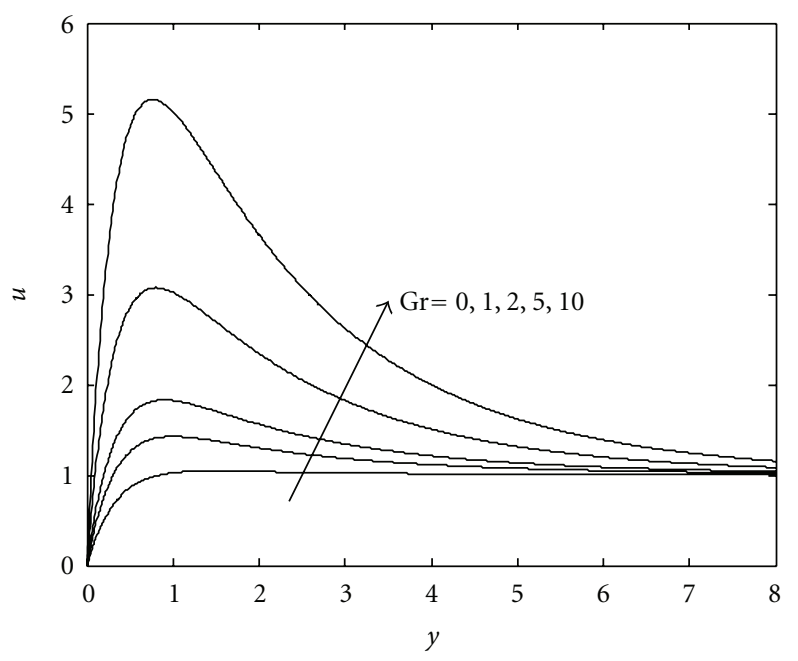

(a)

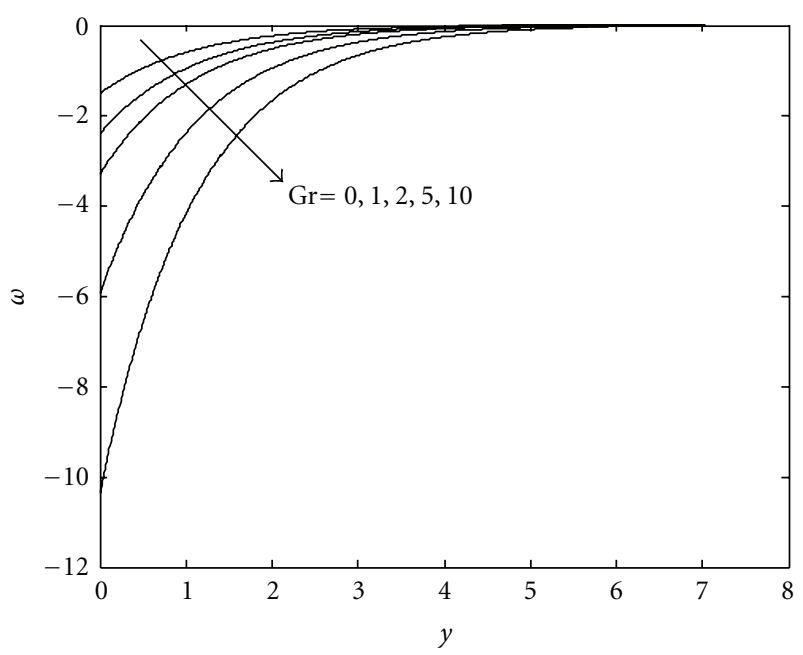

(b)

FIGURE 2: Velocity and microrotation profiles for various values of $\mathrm{Gr}$.

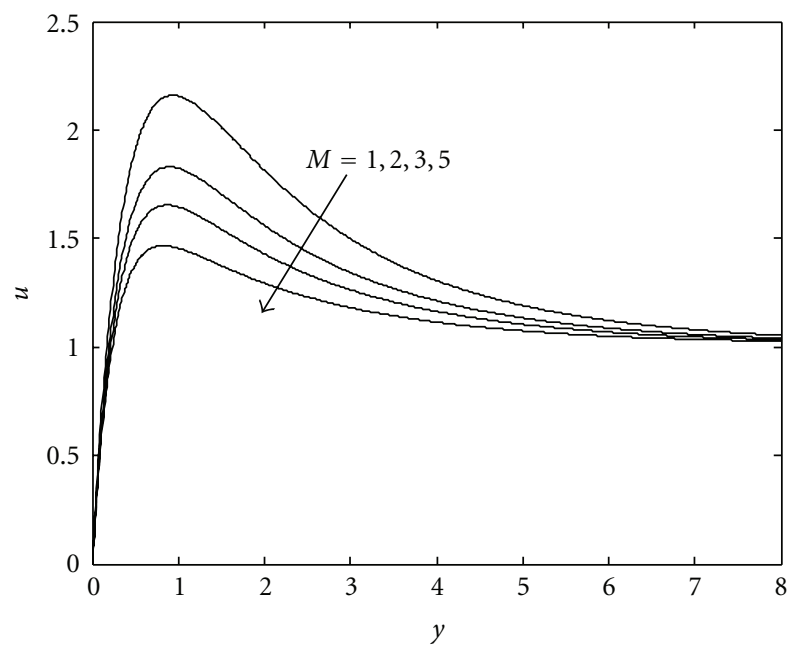

(a)

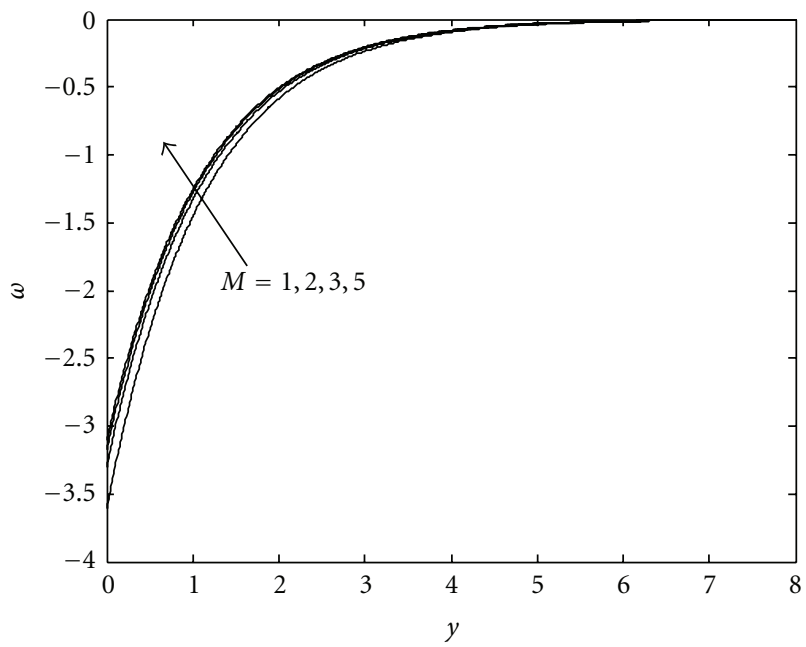

(b)

Figure 3: Velocity and microrotation profiles for various values of $M$. 


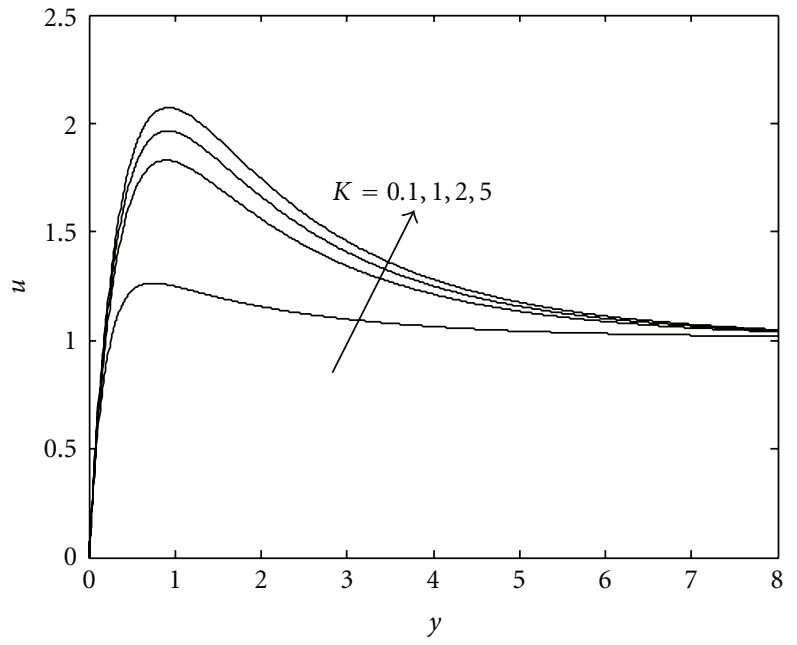

(a)

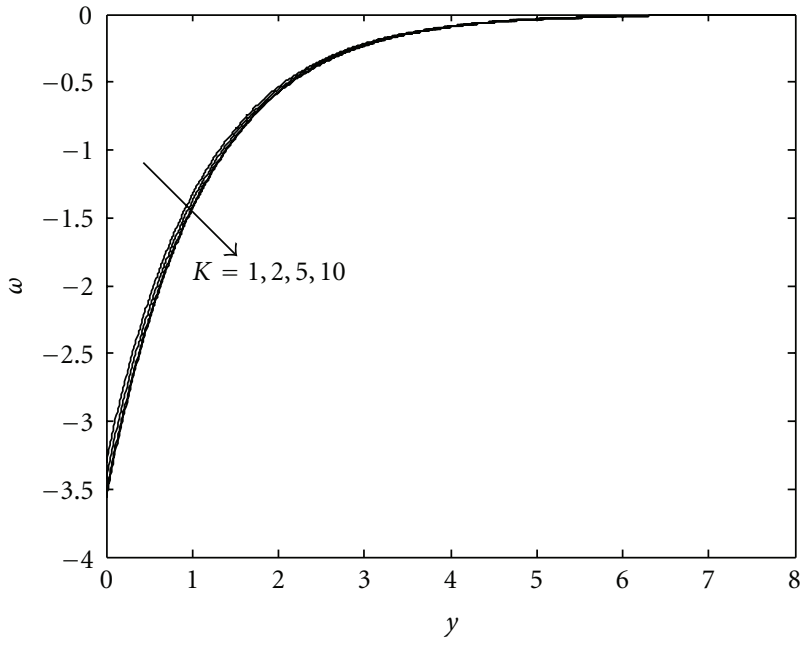

(b)

FIgURE 4: Velocity and microrotation profiles for various values of $K$.

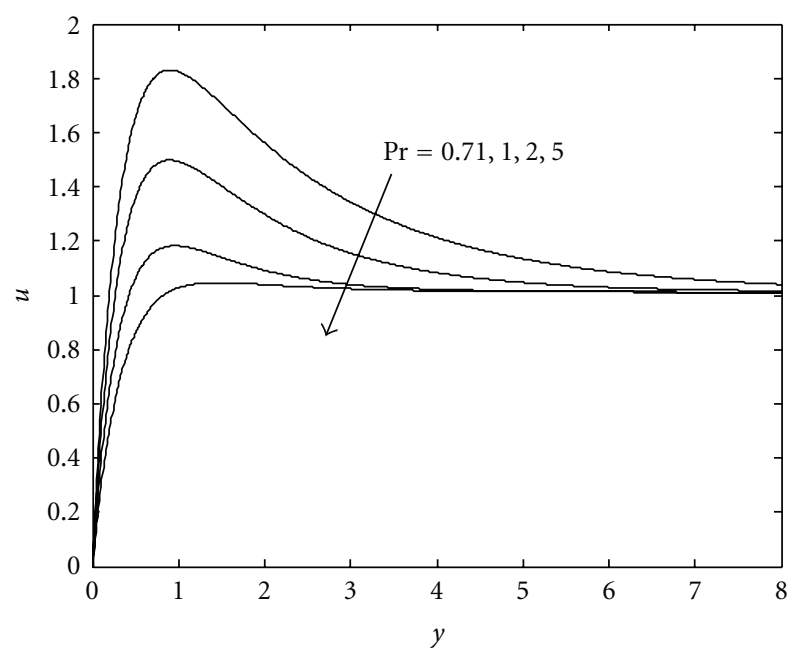

(a)

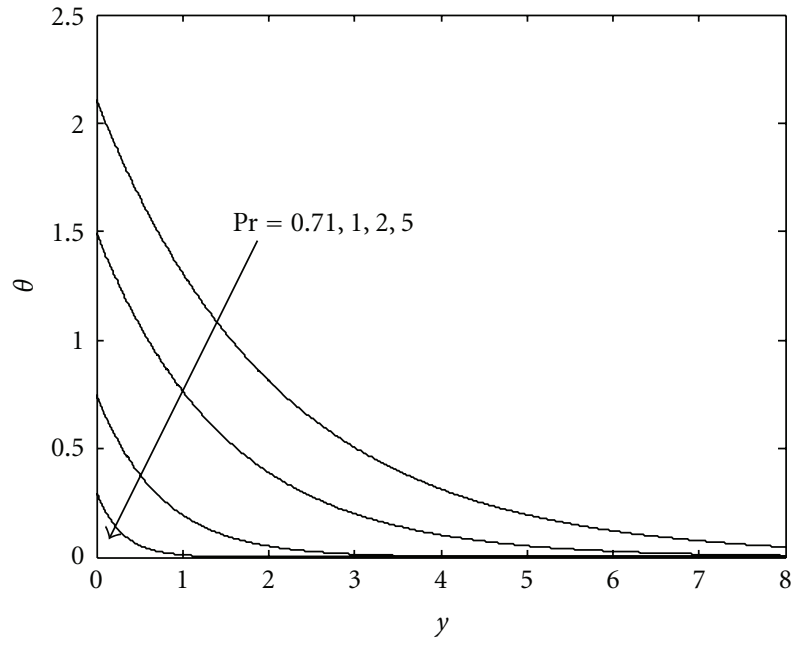

(b)

Figure 5: Velocity and temperature profiles for various values of Pr.

shown Figure 5(b) for different values of Prandtl number Pr. The results show that an increase of Prandtl number results in decreasing the thermal boundary layer thickness and more uniform temperature distribution across the boundary layer. The reason is that smaller values of $\mathrm{Pr}$ are equivalent to increasing the thermal conductivities, and therefore heat is able to diffuse away from the heated surface more rapidly than for higher values of Pr. Hence, the boundary layer is thicker and rate of heat transfer is reduced, as gradients have been reduced.

For various values of the radiation parameter $R$, the velocity and temperature profiles are plotted in Figure 6. It is obvious that an increase in the radiation parameter $R$ results in decreasing velocity and temperature within the boundary layer, as well as a decreased thickness of the velocity and temperature boundary layers. This is because the large $R$-values correspond to an increased dominance of conduction over radiation thereby decreasing buoyancy force (thus, vertical velocity) and thickness of the thermal and momentum boundary layers.

Numerical values for functions proportional to shear stress and wall couple stress are given in Table 1. It is noted that both the skin-friction coefficient and the wall couple stress coefficient decreases as Pr increases. From Table 1, it is noticed that as Gr increases, the skin friction and wall couple stress coefficient increases. It is obvious that an increase in the magnetic field parameter or the radiation parameter reduces the skin friction and wall couple stress coefficient.

\section{Conclusions}

An analysis is presented for the problem of unsteady twodimensional laminar flow of a viscous incompressible micropolar fluid past a vertical porous plate in the presence of a 


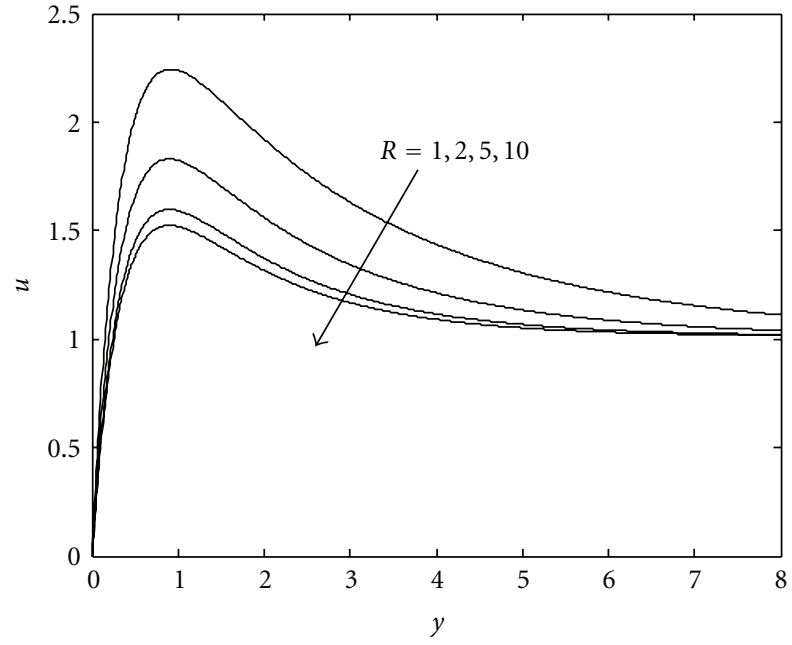

(a)

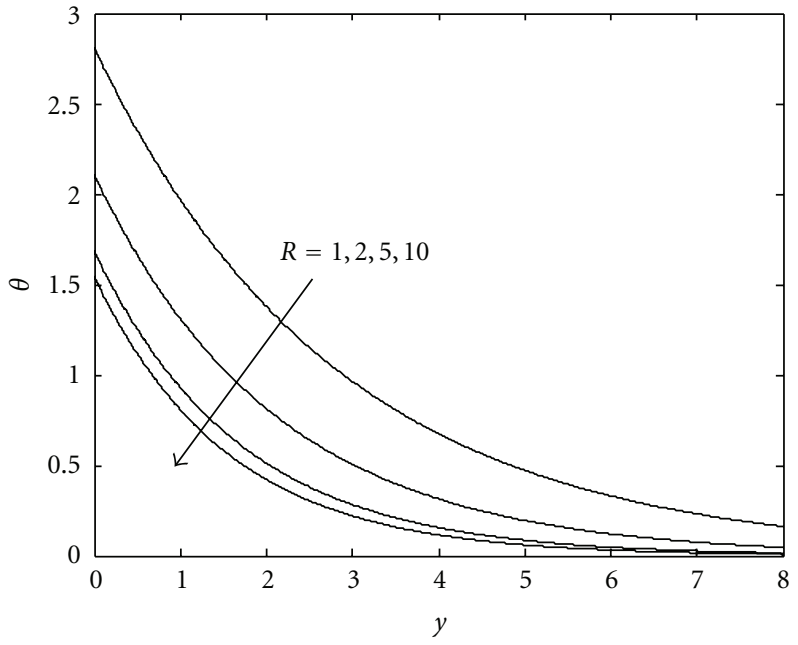

(b)

FIGURE 6: Velocity and temperature profiles for various values of $R$.

TABLe 1: Values of $C_{f}$ and $C_{m}$ for various values of $\mathrm{Gr}, M$, Pr, and $R$ with $\beta=0.2, t=1.0, n=0.1, \varepsilon=0.001, A=0.5, K=1.0$, and $B=0.1$.

\begin{tabular}{cccccc}
\hline Gr & $M$ & $\operatorname{Pr}$ & $R$ & $C_{f}$ & $C_{m}$ \\
\hline 2.0 & 2.0 & 0.71 & 2.0 & 6.9567 & 3.3146 \\
4.0 & 2.0 & 0.71 & 2.0 & 10.5384 & 5.0936 \\
2.0 & 3.0 & 0.71 & 2.0 & 6.7482 & 3.1875 \\
2.0 & 2.0 & 2.0 & 2.0 & 4.5211 & 2.1690 \\
2.0 & 2.0 & 0.71 & 5.0 & 6.1079 & 2.9172 \\
\hline
\end{tabular}

transverse magnetic field and thermal radiation with variable heat flux. Numerical results are presented to illustrate the details of the MHD convective radiative flow and heat transfer characteristics and their dependence on the fluid properties and flow conditions. We may conclude that the translational velocity across the boundary layer and the magnitude of microrotation at the wall are decreased with increasing values of $M$ and $K$. Also, the translational velocity across the boundary layer and temperature at the wall are decreased with increasing values of $M, \operatorname{Pr}$, and $R$, while they show opposite trends with increasing values of Gr.

\section{Appendix}

The exponential indices in (19) are defined by

$$
\begin{gathered}
h_{1}=\frac{\eta}{2}\left[1+\sqrt{1+\frac{4 n}{\eta}}\right], \\
h_{2}=\frac{1}{2(1+\beta)}[1+\sqrt{1+4 N(1+\beta)}], \\
h_{3}=\frac{1}{2(1+\beta)}[1+\sqrt{1+4(N+n)(1+\beta)}], \\
h_{4}=\frac{\Gamma}{2}\left[1+\sqrt{1+\frac{4 n}{\Gamma}}\right],
\end{gathered}
$$

and the coefficients are given by

$$
\begin{aligned}
& a_{1}=-1-a_{2}-a_{3}, \quad a_{2}=-\frac{(\mathrm{Gr} / \Gamma)}{(1+\beta) \Gamma^{2}-\Gamma-N} \\
& a_{3}=\frac{2 \beta \eta}{(1+\beta) \eta^{2}-\eta-N} c_{1} \\
& b_{1}=\frac{2 \beta h_{1} c_{2}}{(1+\beta) h_{1}^{2}-h_{1}-(N+n)}, \\
& b_{2}=\frac{A h_{2} a_{1}}{(1+\beta) h_{2}^{2}-h_{2}-(N+n)}, \quad b_{3}=k_{2}-b_{1} \text {, } \\
& b_{4}=-\frac{\left(\mathrm{Gr} / h_{4}\right)(B+(A \Gamma / n))}{(1+\beta) h_{4}^{2}-h_{4}-(N+n)}, \\
& b_{5}=\frac{A a_{2} \Gamma+(\mathrm{Gr} A / n)}{(1+\beta) \Gamma^{2}-\Gamma-(N+n)}, \\
& b_{6}=\frac{A\left(a_{3} \eta-\left(2 \beta \eta^{2} c_{1} / n\right)\right)}{(1+\beta) \eta^{2}-\eta-(N+n)}, \\
& k_{1}=\frac{A \eta}{n} c_{1}+\frac{1}{2}\left(b_{2} h_{2}+b_{4} h_{4}+b_{5} \Gamma+b_{6} \eta\right), \\
& k_{2}=-\left(1+b_{2}+b_{4}+b_{5}+b_{6}\right), \\
& c_{1}=\left[\frac{(1+\beta) \eta^{2}-\eta-N}{\eta^{2}-\eta-N+\beta h_{2} \eta}\right]\left[\frac{a_{2} \Gamma-h_{2}\left(a_{2}+1\right)}{2}\right] \text {, } \\
& c_{2}=\left[\frac{(1+\beta) h_{1}^{2}-h_{1}-N}{h_{1}^{2}-h_{1}-N+\beta h_{1} h_{3}}\right]\left[k_{1}+\frac{1}{2} k_{2} h_{3}\right] \text {. }
\end{aligned}
$$

\section{Nomenclature}

$A$ : Suction velocity parameter

$B_{0}$ : Magnetic flux density

$B$ : Real positive constant 
$C_{f}:$ Skin-friction coefficient

$C_{m}$ : Couple stress coefficient

$c_{p}$ : Specific heat at constant pressure

$g$ : Acceleration due to gravity

Gr: Thermal Grashof number

$j: \quad$ Microinertia per unit mass

$k$ : Thermal conductivity

$M$ : Magnetic field parameter

$n: \quad$ Dimensionless exponential index

p: Pressure

Pr: Prandtl number

$q_{r}$ : The radiation heat flux

$R: \quad$ Radiation parameter

$t$ : Time

T: $\quad$ Temperature

$u, v$ : Components of velocities along and perpendicular to the plate

$U_{0}$ : Scale of free stream velocity

$V_{0}$ : Scale of suction velocity

$x, y$ : Distances of along and perpendicular to the plate.

\section{Greek Symbols}

$\alpha$ : Fluid thermal diffusivity

$\beta$ : Ratio of vertex viscosity and dynamic viscosity

$\beta_{f}$ : Coefficient of volumetric expansion of the working fluid

$\gamma$ : Spin gradient viscosity

$\delta$ : Scalar constant

$\varepsilon: \quad$ Scalar constant $(\ll 1)$

$\theta$ : Dimensionless temperature

$\Lambda$ : Coefficient of vertex (microrotation) viscosity

$\mu$ : Fluid dynamic viscosity

$\rho$ : Fluid density

$\sigma$ : Electrical conductivity

$v$ : Fluid kinematic viscosity

$v_{r}$ : Fluid dynamic rotational viscosity

$\tau$ : Friction coefficient

$\omega$ : Angular velocity vector.

\section{Subscripts}

$w$ : Wall condition

$\infty$ : Free steam condition.

\section{Superscripts}

()$^{\prime}$ : Differentiation with respect to $y$

*: Dimensional properties.

\section{References}

[1] A. C. Eringen, "Theory of micropolar fluids," Journal of Mathematics and Mechanics, vol. 16, pp. 1-18, 1966.
[2] A. C. Eringen, "Theory of thermomicrofluids," Journal of Mathematical Analysis and Applications, vol. 38, no. 2, pp. 480496, 1972.

[3] G. Ahmadi, "Self-similar solution of imcompressible micropolar boundary layer flow over a semi-infinite plate," International Journal of Engineering Science, vol. 14, no. 7, pp. 639646, 1976.

[4] J. Peddieson and R. P. McNitt, "Boundary layer theory for micropolar fluid," Recent Advances in Engineering Science, vol. 5, pp. 405-426, 1970.

[5] H. S. Takhar and V. M. Soundagekhar, "Flow and heat transfer of a micropolar fluid past a porous plate," Indian Journal of Pure Applied Mathematics, vol. 16, no. 5, pp. 552-558, 1985.

[6] R. S. R. Gorla, "Combined forced and free convection in micropolar boundary layer flow on a vertical flat plate," International Journal of Engineering Science, vol. 26, no. 4, pp. 385-391, 1988.

[7] A. Raptis, "Flow of a micropolar fluid past a continuously moving plate by the presence of radiation," International Journal of Heat and Mass Transfer, vol. 41, no. 18, pp. 2865-2866, 1998.

[8] R. C. Sharma and U. Gupta, "Thermal convection in micropolar fluids in porous medium," International Journal of Engineering Science, vol. 33, no. 13, pp. 1887-1892, 1995.

[9] Y. J. Kim, "Unsteady convetion flow of micropolar fluids past a vertical porous plate embedded in a porous medium," Acta Mechanica, vol. 148, no. 1-4, pp. 105-116, 2001.

[10] Y. J. Kim, "Unsteady MHD convection flow of polar fluids past a vertical moving porous plate in a porous medium," International Journal of Heat and Mass Transfer, vol. 44, no. 15, pp. 2791-2799, 2001.

[11] V. M. Soundalgekar, "Free convection effects on the oscillatory flow past an infinite, vertical, porous plate with constant suction," Proceedings of the Royal Society A, vol. 333, pp. 2536, 1973.

[12] A. A. Raptis, "Flow through a porous medium in the presence of a magnetic field," International Journal of Energy Research, vol. 10, no. 1, pp. 97-100, 1986.

[13] M. A. El-Hakiem, A. A. Mohammadein, S. M. M. El-Kabeir, and R. S. R. Gorla, "Joule heating effects on magnetohydrodynamic free convection flow of a micropolar fluid," International Communications in Heat and Mass Transfer, vol. 26, no. 2, pp. 219-227, 1999.

[14] M. M. Rahman and M. A. Sattar, "Magnetohydrodynamic convective flow of a micropolar fluid past a continuously moving vertical porous plate in the presence of heat generation/absorption," Journal of Heat Transfer, vol. 128, no. 2, pp. 142-152, 2006.

[15] A. Raptis, "Flow of a micropolar fluid past a continuously moving plate by the presence of radiation," International Journal of Heat and Mass Transfer, vol. 41, no. 18, pp. 2865-2866, 1998.

[16] E. M. Abo-Eldahab and A. F. Ghonaim, "Radiation effect on heat transfer of a micropolar fluid through a porous medium," Applied Mathematics and Computation, vol. 169, no. 1, pp. 500-510, 2005.

[17] Y. J. Kim and A. G. Fedorov, "Transient mixed radiative convection flow of a micropolar fluid past a moving, semi-infinite vertical porous plate," International Journal of Heat and Mass Transfer, vol. 46, no. 10, pp. 1751-1758, 2003.

[18] M. Q. Brewster, Thermal Radiative Transfer and Properties, John Wiley \& Sons, New York, NY, USA, 1992. 

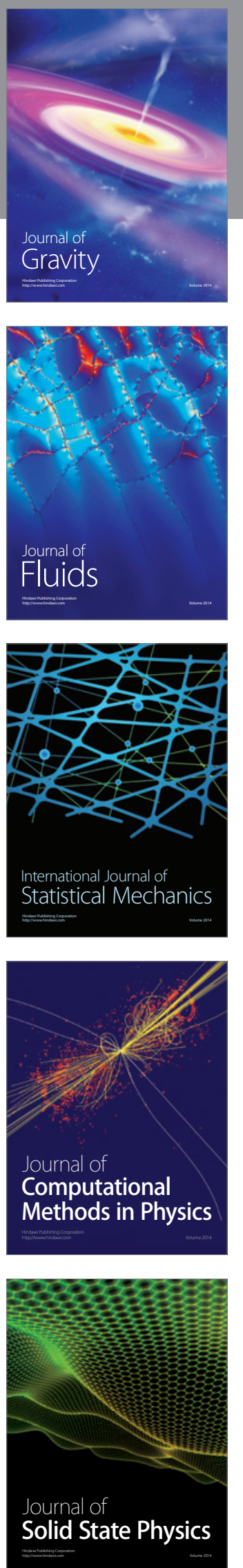
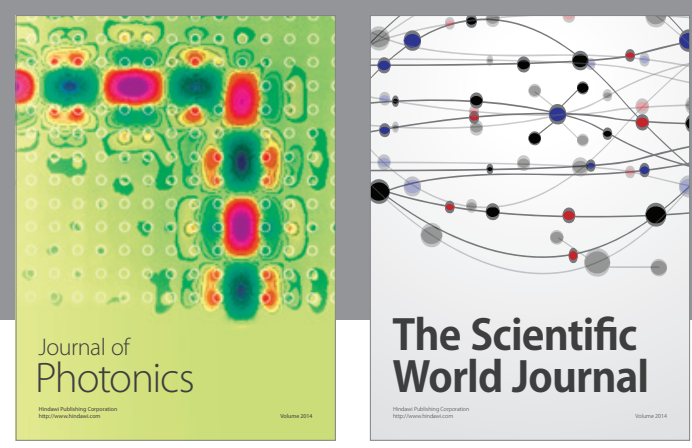

The Scientific World Journal

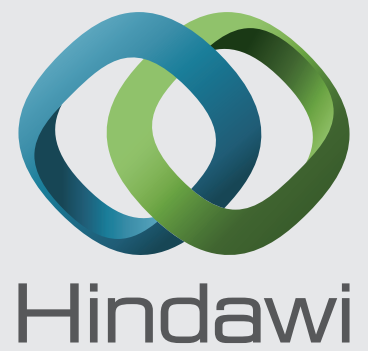

Submit your manuscripts at http://www.hindawi.com
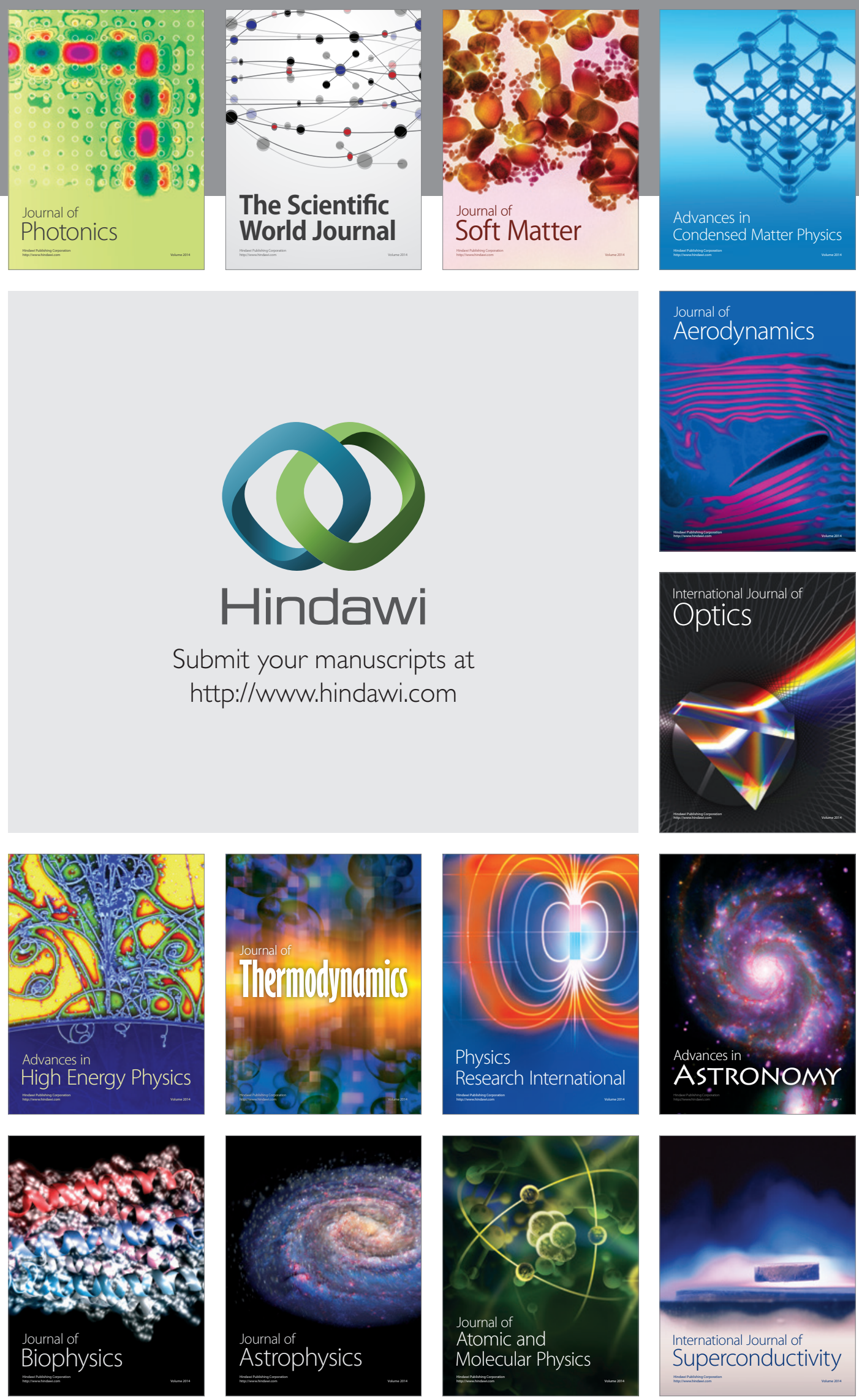
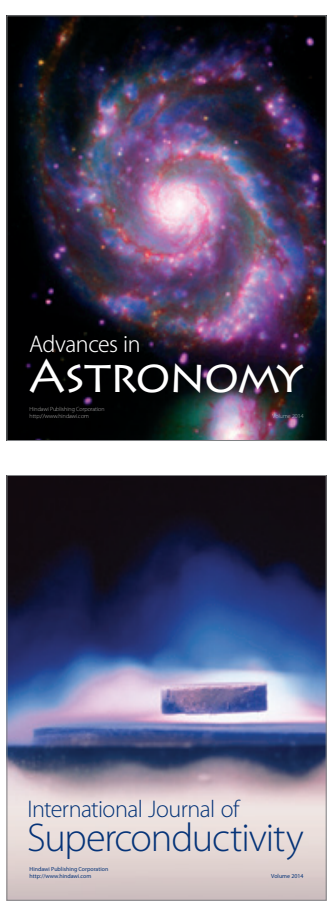